\title{
Linear optimization method for layout of unconstrained damping materials with negligible effect on eigenmode
}

\author{
Seita INOZUME* and Tatsuhito AIHARA** \\ ${ }^{*}$ Hosei University Graduate Schools \\ 3-7-2 Kajinocho, Koganei-shi, Tokyo 184-8584, Japan \\ E-mail: seita.inozume.5z@stu.hosei.ac.jp \\ **Hosei University \\ 3-7-2 Kajinocho, Koganei-shi, Tokyo 184-8584, Japan
}

Received: 1 March 2020; Revised: 17 August 2020; Accepted: 1 October 2020

\begin{abstract}
Damping materials are sometimes employed to reduce vibrations. In vehicles, weight reduction and a high damping effect are required to reduce environmental impacts. Many studies have been conducted to determine an optimal topological layout with a high damping effect under a limited amount of damping material. However, many methods require finite element analysis to be performed several times owing to the nonlinearity of the objective function. In addition, if there exists a problem with the optimal shape, it is necessary to use a filter or change the penalty parameter and perform optimization again. Therefore, this study proposes a method to linearize optimization problems to reduce the number of finite element analysis procedures required, under the assumption that the layout of the damping material does not affect the eigenmode when the base structure is damped via unconstrained layer damping treatment. Several numerical examples are provided to demonstrate the effectiveness of proposed method. The verification results show that the optimal layout and loss factor of the proposed and conventional methods were similar for a large-scale finite element analysis model. Moreover, this study proposes an optimality evaluation method using the modal assurance criterion. As long as the assumption holds true, the proposed method can significantly improve the time efficiency when repeating optimization under different conditions.
\end{abstract}

Keywords: Topology optimization, Damping material, Loss factor, Modal strain energy method, Finite element method

\section{Introduction}

In automobiles and railway vehicles, it is necessary to reduce vibrations and noise to improve the comfort within the vehicle; one of the methods to achieve this is to use damping materials. On the other hand, it is also desirable to reduce the amount of damping material used from the viewpoint of weight reduction, environmental load, and cost. One way to obtain a design that satisfies these requirements is to use topology optimization (Bendsøe and Kikuchi, 1988). Topology optimization is the most flexible design optimization method and creates highest performance shapes with limited materials. Methods for expressing the presence or absence of a material include arranging design variables for each element and node as per the finite element method. In addition, various problems have also been investigated (Ma et al., 1995; Du and Olhoff., 2007).

A property called the loss factor is used as an index for evaluating the damping performance of damping materials. The loss factor is considered in the cases of certain damping mechanisms, namely, structural damping and hysteretic damping; here, the magnitude of the damping does not depend on the strain rate. Moreover, not only the material properties but also the damping of a complex system can be expressed. However, when the loss factor of the entire system is calculated using an arbitrary model with structural damping according to the finite element method, the components of the stiffness matrix required for calculation are complex numbers; therefore, it is necessary to perform complex eigenvalue analysis, and the calculation load becomes high. 
Another way to calculate the loss factor is to use the modal strain energy (MSE) method instead of performing a complex eigenvalue analysis (Johnson and Kienholz, 1982). According to this method, the modal loss factor is expressed as the strain energy sharing ratio of the damping material to the entire system in a certain mode, multiplied by the loss factor of the damping material. As the eigenvectors obtained via real eigenvalue analysis are used to calculate the strain energy, the computational load is smaller than that of complex eigenvalue analysis; however, the accuracy decreases when the eigenvector changes due to attenuation are large. Many studies have been conducted to apply topology optimization in the calculation of the loss factor using the MSE method to create the optimal layout of damping materials. Yamamoto et al. (2014) exploited the convenience of general structural analysis codes to approximate the modal loss factor using the MSE method with real eigenvalues and optimized the layout with unconstrained damping materials using the modal loss factor as the objective function. In addition, a distance-weighted averaging filter was used for design sensitivity to combine the damping material areas into one continuous material to reduce manufacturing costs. However, to achieve a continuous damping material, it is necessary to repeat the optimization calculation to obtain the required filter radius, whereby the calculation time greatly increases particularly for a large analysis model. Ling et al. (2011) aimed to calculate the modal loss factor using the MSE method using a finite element that can express a flat plate, damping material, and constrained layer as one element and subsequently maximize it. The calculation of design sensitivity relies on the assumption that mode changes can be ignored; however, as this applies only to the extent of small changes in design variables, the calculation is iterative. As the number of iterations exceeds 100 by the time of convergence and eigenvalue analysis is included in the iterative calculation, it is considered that the time efficiency becomes extremely poor when the analysis model is large.

In this study, we propose a method for linearizing the optimization problem under the assumption that mode changes are negligible regardless of the design variable, when the rigidity and mass of the damping material are smaller than those of the base structure, to improve the time efficiency of topology optimization. Furthermore, to show the effectiveness of the method, a finite element model was created and compared with the conventional method. An optimality evaluation method is also proposed using a modal assurance criterion to show that optimality of the optimization result using this method is not impaired. In addition, an optimization was performed for multiple modes to show that the application range of this method is effective for multiple modes.

\section{Maximization of modal loss factor}

\subsection{Modal strain energy method}

This study considered a system consisting of two kinds of materials, namely, a damping material and a base structure whose damping can be ignored. Structural damping can be expressed by adding complex stiffness, so the stiffness of the system including the structural damping material can be expressed as complex stiffness. According to the MSE method, the modal loss factor $\eta_{i}$ in the $i$ th mode is expressed as follows.

$$
\eta_{i}=\frac{\left(\boldsymbol{\phi}_{R i}\right)^{T} \boldsymbol{K}_{I} \boldsymbol{\phi}_{R i}}{\left(\boldsymbol{\phi}_{R i}\right)^{T} \boldsymbol{K}_{R} \boldsymbol{\phi}_{R i}}
$$

where $\boldsymbol{K}_{R}$ and $\boldsymbol{K}_{I}$ are the real and imaginary parts of the system stiffness matrix, respectively, and $\boldsymbol{\phi}_{R i}$ is the real eigenvector in the $i$ th mode. The stiffness of the entire system $\boldsymbol{K}_{R}$ can also be expressed in terms of the damping material stiffness $\boldsymbol{K}_{R d}$ and the base structure stiffness $\boldsymbol{K}_{R p}$, as shown in Eq. (2).

$$
\boldsymbol{K}_{R}=\boldsymbol{K}_{R d}+\boldsymbol{K}_{R p}
$$

Finally, the complex stiffness $\boldsymbol{K}$ of the entire system can be expressed as follows.

$$
\boldsymbol{K}=\boldsymbol{K}_{R}+j \boldsymbol{K}_{I}=\left(\boldsymbol{K}_{R p}+\boldsymbol{K}_{R d}\right)+j \eta_{m} \boldsymbol{K}_{R d}
$$

where $\eta_{m}$ is the loss factor of the damping material. Using Eq.(2) and (3), Eq. (1) can be rewritten as follows. 


$$
\eta_{i}=\frac{\left(\boldsymbol{\phi}_{R i}\right)^{T} \boldsymbol{K}_{R d} \boldsymbol{\phi}_{R i}}{\left(\boldsymbol{\phi}_{R i}\right)^{T}\left(\boldsymbol{K}_{R p}+\boldsymbol{K}_{R d}\right) \boldsymbol{\phi}_{R i}} \eta_{m}
$$

Therefore, the modal loss factor can be expressed as the product of the strain energy distribution rate of the damping material and the loss factor of the damping material. Furthermore, if the strain energy of the damping material in the $i$ th mode is assumed to be lower than the strain energy of the vibration of the base structure, Eq. (4) can be expressed as follows.

$$
\eta_{i} \approx \frac{\left(\boldsymbol{\phi}_{R i}\right)^{T} \boldsymbol{K}_{R d} \boldsymbol{\phi}_{R i}}{\left(\boldsymbol{\phi}_{R i}\right)^{T} \boldsymbol{K}_{R p} \boldsymbol{\phi}_{R i}} \eta_{m}
$$

The proposed method maximizes the modal loss factor using Eq. (5).

\subsection{Density method}

Numerous methods have been proposed for expressing the material distribution in topology optimization; these include the homogenization method (Suzuki and Kikuchi, 1991) and the level set method (Wang et al., 2003). In this study, the density method (Yang and Chuang, 1994), which is often employed in general-purpose finite element analysis software, was used because it can be easily implemented in programs. The density method produces grayscale (Sigmund, 2007) unlike the level set method; however, the amount is often small, and this method can be used in engineering practice by subsequently processing the optimization result (Alok et al., 2014; Ercan et al., 2015). The optimization example presented in this report was also confirmed to exhibit a small amount of grayscale, and hence there is no issue in this regard. The elastic tensor $\boldsymbol{E}$ of a material with elastic tensor $\boldsymbol{E}_{\mathbf{0}}$ can be expressed as a function of the normalized density $\rho$ only.

$$
\boldsymbol{E}=f(\boldsymbol{\rho}) \boldsymbol{E}_{\mathbf{0}}, \quad 0 \leq \boldsymbol{\rho} \leq 1
$$

The relationship between the presence or absence of a material and the material properties can be expressed by defining the function $f(\boldsymbol{\rho})$, such that a normalized density of 1 indicates the presence of the material while a normalized density of 0 indicates the absence of the material. An equation based on the solid isotropic material with penalization (SIMP) method (Bendsøe and Sigmund, 1999) is often used for this function, which is expressed as follows.

$$
f(\boldsymbol{\rho})=\boldsymbol{\rho}^{p}
$$

where $p$ is a penalty parameter. If this penalty parameter is set to 1 , the elastic modulus changes linearly with the normalized density, and hence the normalized density converges at an intermediate density such as 0.5 called "grayscale" during optimization, and the resulting shape cannot be interpreted in an engineering manner. Upon introduction of a volume constraint in the optimization problem, through formulation of the effective volume as proportional to the normalized density, the penalty parameter is set to 2 or 3 to set the stiffness per effective volume at the intermediate density. Through intentional lowering of the stiffness per effective volume at the intermediate density, convergence at intermediate densities can be avoided, and a clear structure can be obtained.

In this study, a normalized density was assigned to each element in the design domain as a design variable. In addition, when the design variable was 0 , the stiffness was set to a small value instead of 0 to avoid instability in numerical calculations,. Thus, the design variable $\rho_{k}$ was assigned to the $k$ th design domain element, and the Young's modulus of the design domain element $E_{k}$ was expressed as follows.

$$
E_{k}=\rho_{k}^{p} E_{m}+E_{\text {min }}
$$

where $E_{m}$ and $E_{\min }$ are the Young's modulus of material and the small value, respectively. The small constant $E_{\min }$ is used to avoid numerical instability, and a much smaller value than the Young's modulus is chosen when the normalized density is 1 . The same property interpolation method is applied to mass density, and other material properties, such as Poisson's ratio and the loss factor, are fixed, regardless of the design variables. 


\subsection{Optimization problem}

The optimization problem was formulated as follows.

Minimize $F=-\eta_{i}$

$$
\text { Subject to }\left\{\begin{array}{l}
\left(\boldsymbol{K}_{R}-\omega_{i}{ }^{2} \boldsymbol{M}\right) \boldsymbol{\phi}_{R i}=0 \\
\sum_{k=1}^{n} V_{k} \rho_{k} \leq \alpha V_{\text {total }} \\
0 \leq \rho_{k} \leq 1,(k=1,2, \ldots n)
\end{array}\right.
$$

where $\omega_{i}$ is the $i$ th natural angular frequency, $\boldsymbol{M}$ is the mass matrix, $V_{k}$ is the volume of $k$ th element, $\alpha$ is the volume constraint ratio, and $V_{\text {total }}$ is the total volume of design domain. The modal loss factor was maximized by minimizing the negative value of the modal loss factor in Eq. (5), which served as the objective function. When the modal loss factor for multiple modes was maximized, the objective function in Eq. (9) was replaced with a simple sum as shown in Eq. (13).

$$
\operatorname{Minimize} F=-\sum_{i} \eta_{i}
$$

As the optimization problem was nonlinear, design sensitivity calculations were used to solve it. Assuming that the change in the eigenmode was negligible for a small change in the design variables, the design sensitivity of the modal loss factor in Eq. (5) was expressed as follows.

$$
\frac{\partial \eta_{i}}{\partial \rho_{k}}=\frac{\left(\boldsymbol{\phi}_{R i}\right)^{T} \frac{\partial \boldsymbol{K}_{R d}{ }^{\prime}}{\partial \rho_{k}} \boldsymbol{\phi}_{R i}}{\left(\boldsymbol{\phi}_{R i}\right)^{T} \boldsymbol{K}_{R p} \boldsymbol{\phi}_{R i}} \eta_{m}
$$

where $\boldsymbol{K}_{R d}{ }^{\prime}$ is the overall stiffness matrix of the damping material at an arbitrary design variable and can be written as follows.

$$
\boldsymbol{K}_{R d}{ }^{\prime}=\sum_{k=1}^{n} \rho_{k}{ }^{p} \boldsymbol{K}_{R d k}^{e}
$$

where $\boldsymbol{K}_{R d k}^{e}$ is the $k$ th element of the real stiffness matrix of the damping material. For optimization problems with strong non-linearity, such as stiffness maximization problems, a linear approximation was performed using the sensitivity shown in Eq. (14). However, in cases where changes in eigenmodes could be assumed negligible for a range of values for all design variables, the nonlinearity was not considered. Practically, it is applied when the unconstrained damping material is applied and the rigidity and mass of the damping material are sufficiently smaller than those of the base structure. It should be noted that this condition may not hold depending on the order of the eigenmode of interest and the boundary conditions. Therefore, if the penalty parameter in Eq. (15) is set to 1, the modal loss factor can be expressed by the linear sum of design variables as follows.

$$
\eta_{i}=\frac{\eta_{m}}{\left(\boldsymbol{\phi}_{R i}\right)^{T} \boldsymbol{K}_{R p} \boldsymbol{\phi}_{R i}} \sum_{k=1}^{n} \rho_{k}^{p}\left(\boldsymbol{\phi}_{R i}\right)^{T} \boldsymbol{K}_{R d k}^{e} \boldsymbol{\phi}_{R i}
$$

Furthermore, for investigating the change in the optimal result due to the assumptions employed, conventional optimization was performed using the loss factor described in Eq. (4). If the only assumption employed was that the change in the eigenmode is negligible only within the small change in the design variable, the optimization problem became nonlinear, and the required design sensitivity was expressed as 
$\frac{\partial \eta_{i}}{\partial \rho_{k}}=\frac{\left(p \rho_{k}{ }^{(p-1)}\left(\boldsymbol{\phi}_{R i}\right)^{T} \boldsymbol{K}_{R d k}^{e} \boldsymbol{\phi}_{R i} \eta_{m}\right)\left(\left(\boldsymbol{\phi}_{R i}\right)^{T} \boldsymbol{K}_{R} \boldsymbol{\phi}_{R i}\right)-\left(\left(\boldsymbol{\phi}_{R i}\right)^{T} \boldsymbol{K}_{R d} \boldsymbol{\phi}_{R i} \eta_{m}\right)\left(p \rho_{k}{ }^{(p-1)}\left(\boldsymbol{\phi}_{R i}\right)^{T} \boldsymbol{K}_{R d k}^{e} \boldsymbol{\phi}_{R i}\right)}{\left(\left(\boldsymbol{\phi}_{R i}\right)^{T} \boldsymbol{K}_{R} \boldsymbol{\phi}_{R i}\right)^{2}}$

\subsection{Algorithm}

The algorithms that solved the optimization problems in the proposed and conventional methods are shown in Fig. 1 and Fig. 2, respectively. For solving the nonlinear optimization problems shown in Eqs. (9) to (12), the sensitivities shown to approximate a linear programming problem with a small change in the design variables were employed. Thus, it was necessary to solve the optimization problem iteratively. However, as the proposed method allows for linear approximation within the range of changes in all design variables, the final design could be obtained by solving the linear programming problem only once. In this method, the eigenvalue analysis was performed after the design variables were initialized, the coefficients were obtained using Eq. (16), and the optimization was performed. Several methods for topology optimization require convergence judgment and iterative calculation as shown in Fig. 2; however, both these aspects can be omitted in proposed method.

\section{Optimization example using FEM model of a box 3.1 Analysis and optimization settings}

In this method, the optimization flowchart did not include iterations, and the eigenvalue analysis was performed only once, which is advantageous for large-scale models. Thus, a case where the damping material was attached to the box shape, as in the finite element analysis model shown in Fig. 3, was considered. The dimensions of the box to be damped were 200, 250, and $250 \mathrm{~mm}$ in the $\mathrm{x}$, y, and $\mathrm{z}$ directions, respectively, as shown in Fig. 3. The thickness of each face was $1 \mathrm{~mm}$. The displacement boundary condition was that the surface in the negative direction of the $\mathrm{z}$-axis was completely constrained and the damping material domain was defined on the remaining five surfaces with a thickness of $2 \mathrm{~mm}$.

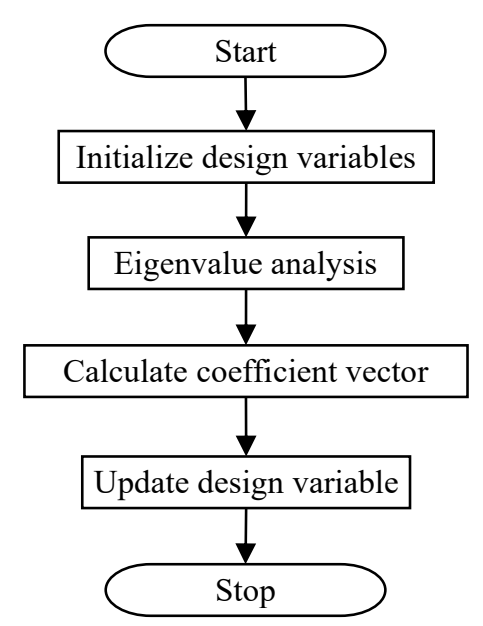

Fig. 1 Flowchart of the proposed optimization process. First, the design variable initialization makes all design variables equal to the volume constraint ratio. Next, eigenvalue analysis is performed, and the results are used to calculate the coefficients of design variables. Finally, design variables are updated via linear programming. 


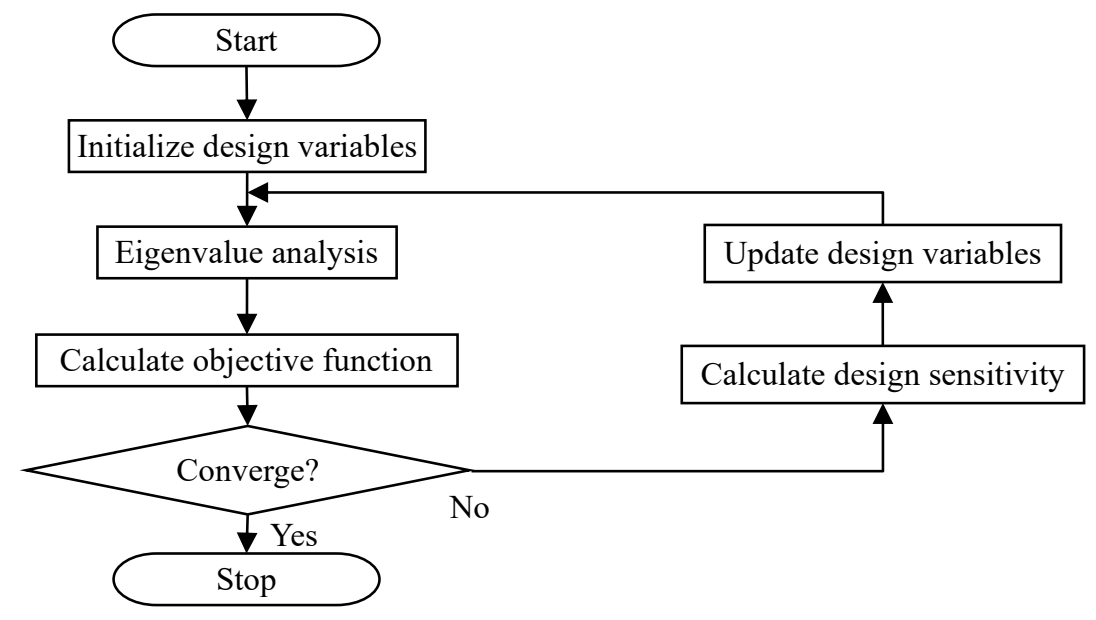

Fig. 2 Flowchart of conventional optimization process. First, the design variable initialization makes all design variables equal to the volume constraint ratio. Next, eigenvalue analysis is performed, and the objective function is calculated using the result. If the objective function converges, the process ends. If not, the design sensitivity is calculated, and the design variables are updated via linear programming.

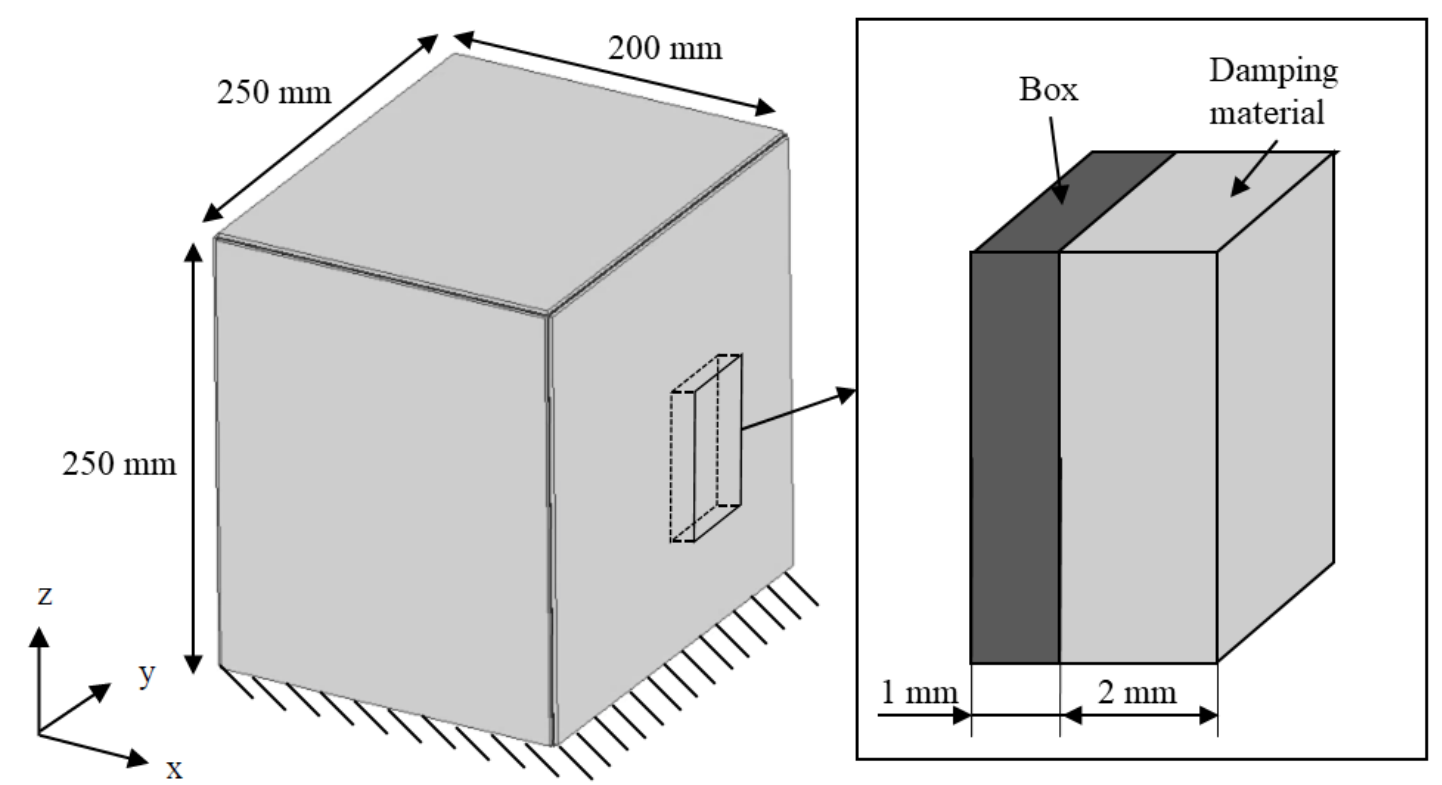

Fig. 3 The finite element model of the box with the design domain for the damping material. The thickness of the box is $1 \mathrm{~mm}$ and the dimensions are 200, 250, and $250 \mathrm{~mm}$ in the $x$-, $y$-, and $z$-directions. The face in the $z$ axis negative direction is constrained, and the design domain for the damping material is defined on the remaining five faces as $2 \mathrm{~mm}$ in thickness.

A 20-node hexahedral quadratic element for each element was used. The material properties of the box and the damping material are shown in Table 1. However, all material properties are constant, regardless of vibration frequency. Furthermore, as described in the previous section, it is necessary to set a small value for the material properties of the design domain for the damping material whose design variable is 0 . The Young's modulus and mass density as the small value were set to $10^{-3} \mathrm{~Pa}$ and $10^{-9} \mathrm{~kg} / \mathrm{m}^{3}$, respectively. 
The volume constraint in the optimization was limited to $60 \%$ of the design area; in other words, approximately 0.5 $\mathrm{kg}$ of the total mass of the damping material when the damping material is applied to the entire design domain is limited to approximately $0.3 \mathrm{~kg}$, which is $60 \%$ of the total mass. The initial values of all design variables were set to 0.6 by initializing the design variables in the flowcharts shown in Figs. 1 and 2. In addition to linear programming for optimization in the proposed method, sequential linear programming was also used for optimization in the conventional method. The variation range of the design variables in each step was set to 0.1 . The penalty parameter in Eq. (17) was set to 2 . The linear programming and eigenvalue analysis used in both methods were all performed using MATLAB.

\subsection{Optimal layout}

Optimal layout and mode shape of the damping material after the optimization of the 1st mode using the proposed method is shown in Fig. 4. The parts shown in black represent the areas wherein the damping material was arranged; the parts in white represent the areas wherein the damping material was not arranged. In addition, as the FEM model is a parallelepiped, all displacements and optimal layouts were almost the same as those of the facing surface. The optimal layout had a clear layout with almost no gray scale and no fine layout like a checkerboard (Cameron, 2009), hence, it is suitable for engineering applications. It can be seen from Fig. 4 that most of the damping material is attached to the part where the displacement is large. This is due to the property of the damping material that dissipates energy by deforming.

Table 1 Material properties of the box and damping material.

\begin{tabular}{lcccc} 
& Young's modulus [GPa] & Poisson ratio [-] & Density $\left[\mathrm{kg} / \mathrm{m}^{3}\right]$ & Loss factor [-] \\
\hline Box & 210 & 0.3 & 7860 & 0 \\
Damping material & 0.8 & 0.47 & 920 & 0.4 \\
\hline
\end{tabular}

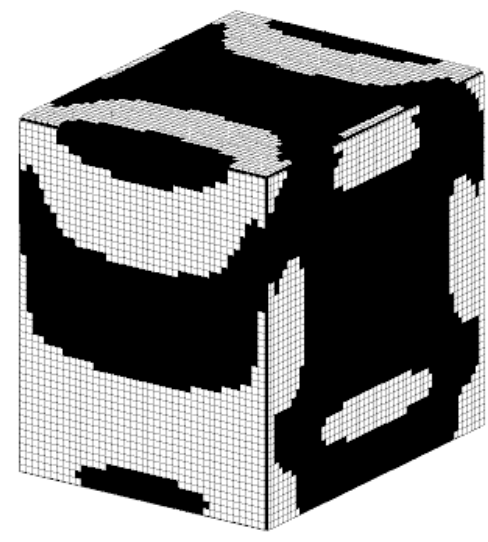

(a)

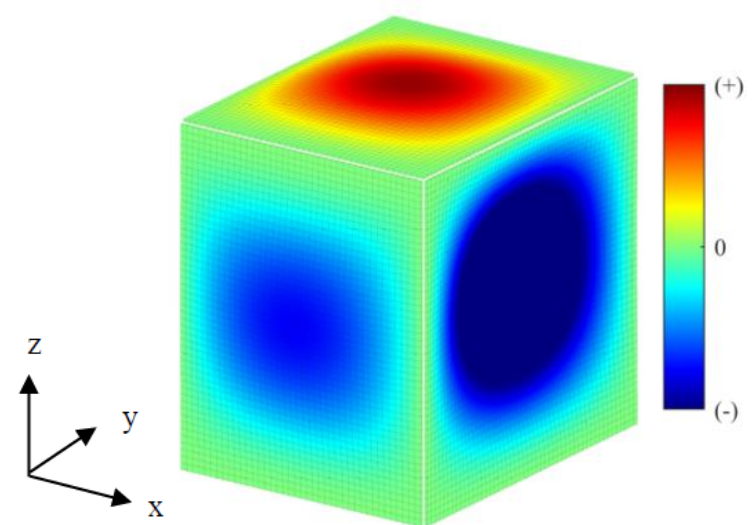

(b)

Fig. 4 (a) Optimal layout and (b) displacement at 1st eigenmode using the proposed method. Optimal layout shows that there were damping materials in the black areas and no damping materials in the white areas. The sign of the displacement map for each face is determined by the axis perpendicular to the face. 


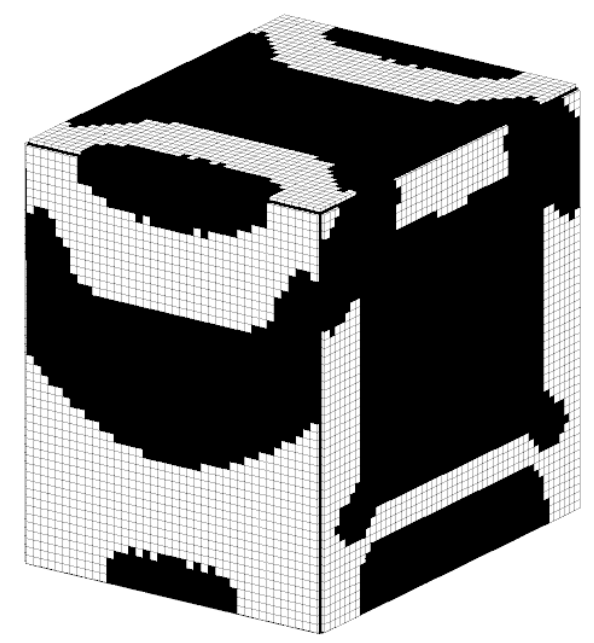

Fig. 5 Optimal layout at 1 st eigenmode using the conventional method.

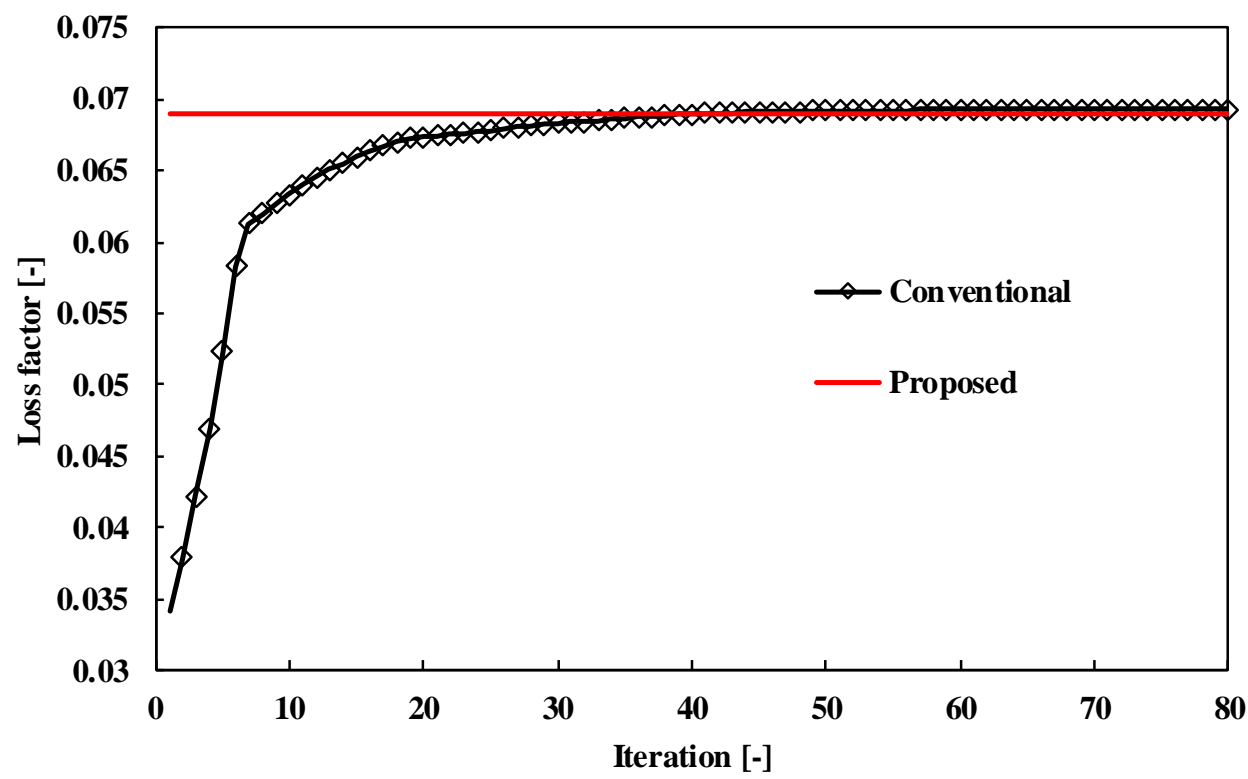

Fig. 6 Iteration histories of optimization at 1st eigenmode. The loss factor determined as per the proposed method is indicated by a red line, while the loss factor at each iteration using the conventional method is indicated by a black line with the marker.

The optimal layout from the conventional method and the change in the modal loss factor of the 1st mode with repetition in the conventional method are shown in Fig. 5 and Fig. 6. Compared to Fig. 4, there was no significant difference in the optimal layout and there was also no grayscale in the conventional method. The modal loss factor attained the value as per this method in approximately 40 iterations, as shown in Fig. 6, and then increased slightly. The final modal loss factor obtained in this method was almost the same as that of the proposed method, with a difference of approximately $0.62 \%$. From these results, it is considered that the change in the mode shape due to the layout of the damping material is insignificant even if the design variable changes substantially; even if there is a change, the effect on the loss factor is very small.

The optimal layout and mode shapes as per the proposed method for the 2nd-5th mode are shown in Figs. 7 to 10 , respectively. Fig. 4 and Figs. 7 to 10 show that the damping material tends to be applied at locations where there are large amount of displacement, regardless of mode orders; however, detailed layouts that were difficult to be estimated from the displacement were also obtained. Some mode orders, such as the 2nd mode x-plane, contained small areas where no damping material was located, although these could be removed using a filter (Sigmund, 2007). 


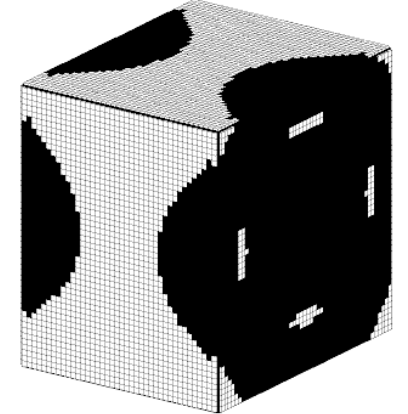

(a)
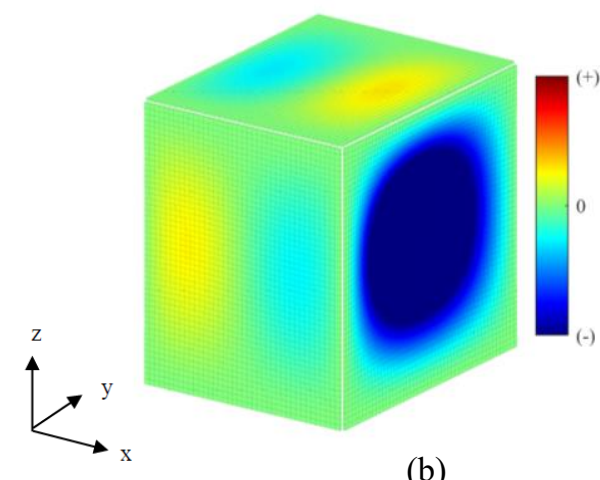

(b)

Fig. 7 (a) Optimal layout and (b) displacement at 2nd eigenmode using the proposed method.

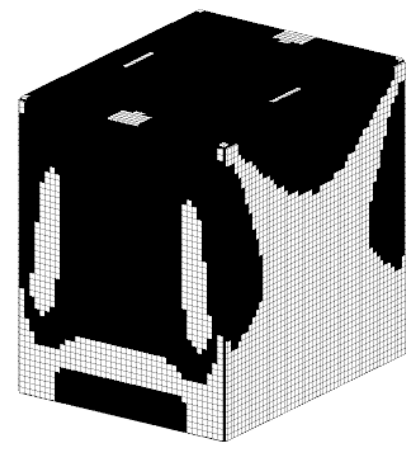

(a)

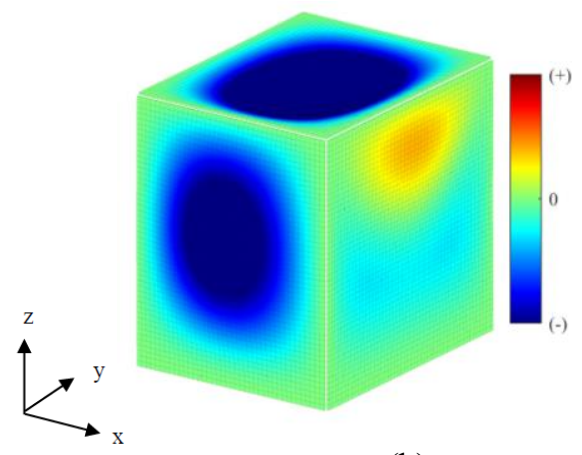

(b)

Fig. 8 (a) Optimal layout and (b) displacement at 3rd eigenmode using the proposed method.

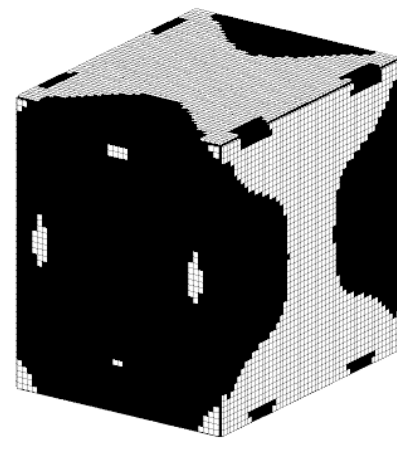

(a)

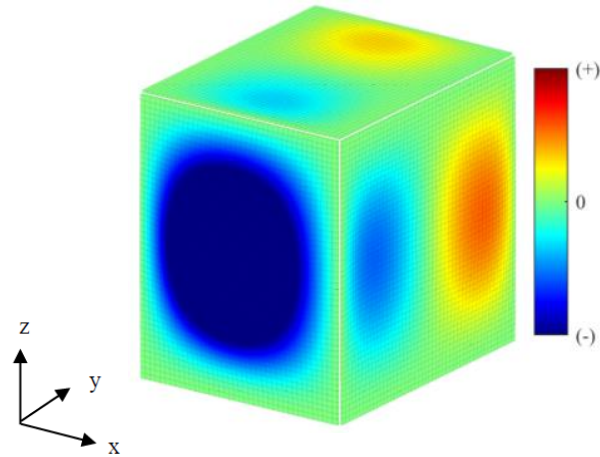

(b)

Fig. 9 (a) Optimal layout and (b) displacement at 4th eigenmode using the proposed method.

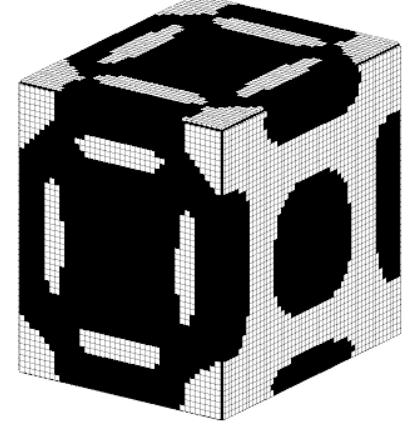

(a)

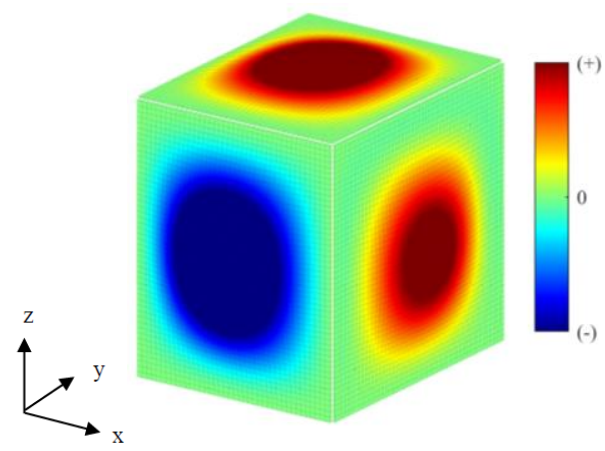

(b)

Fig. 10 (a) Optimal layout and (b) displacement at 5th eigenmode using the proposed method. 


\subsection{Modal Assurance Criterion}

The modal assurance criterion (MAC) (Pastor and Binda, 2012) was used to show the optimality reduction resulting from the implementation of the assumption that the eigenmode changes as per the proposed method can be neglected within the range of possible values of all design variables. MAC is sometimes used to compare the results of the experimental modal analysis and eigenvalue analysis and to check for mode switching when a design is changed. As the MAC value was close to 1 , it indicates that the mode shapes were consistent and the assumption could be considered valid. The calculated MAC values for the initial state in the optimization of the 1st mode and for the eigenmodes from the 1 st to the 5 th modes after optimization are shown in Table 2. The assumption was valid because the component in the first row and first column, which is considered the MAC value, is very close to 1 . The values of the other diagonal components are also close to 1 indicating that there was no significant mode change before and after optimization and that no mode switching had occurred. The same material properties and optimization conditions as described earlier were employed, and the 1st-5th modes were the targets. The results indicate that the solution to the linear programming problem in this optimization model also demonstrated near-optimality, similar to the solution of the original nonlinear programming problem.

\subsection{Optimization result of multiple modes}

For structures that have multiple natural frequencies for the frequency band, optimization for multiple modes is necessary to improve vibration suppression performance. The effectiveness of the proposed method was verified by calculating the simple sum of the coefficient and sensitivity in Eq. (16) and (17) to serve as the objective function of the proposed and conventional methods over multiple modes and performing optimization.

The optimal layout of each method is shown in Fig. 11. The optimal layout between the two methods had slight difference, consistent with the result from the single-mode optimization.

The differences in the modal loss factors for the $1 \mathrm{st}-5$ th modes between the proposed method and the conventional method are shown in Table 3. The loss factors from the proposed method for the 1st, 2nd, and 5th modes were lower than those from the conventional method; however, for the other modes, they were higher than those from the conventional method. In general, although the values from the proposed method were slightly smaller than those from the conventional method, the differences were small; hence the results from the two methods were equivalent.

Table 2 Modal assurance criterion of the design domain for the damping material.

\begin{tabular}{|c|c|c|c|c|c|c|}
\hline & \multicolumn{5}{|c|}{ Initial mode } \\
\hline & & $1 \mathrm{st}$ & 2nd & $3 \mathrm{rd}$ & 4 th & 5 th \\
\hline \multirow{5}{*}{$\begin{array}{l}\text { Mode after } \\
\text { optimization }\end{array}$} & $1 \mathrm{st}$ & 0.99997 & 0.00000 & 0.00001 & 0.00000 & 0.00024 \\
\hline & 2nd & 0.00000 & 0.99996 & 0.00000 & 0.00000 & 0.00000 \\
\hline & $3 \mathrm{rd}$ & 0.00000 & 0.00000 & 0.99970 & 0.00000 & 0.00000 \\
\hline & 4 th & 0.00000 & 0.00000 & 0.00000 & 0.99970 & 0.00000 \\
\hline & 5 th & 0.00000 & 0.00000 & 0.00012 & 0.00000 & 0.99964 \\
\hline
\end{tabular}




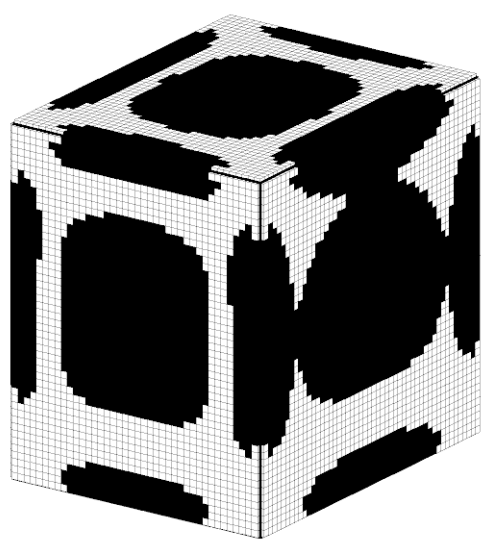

(a)

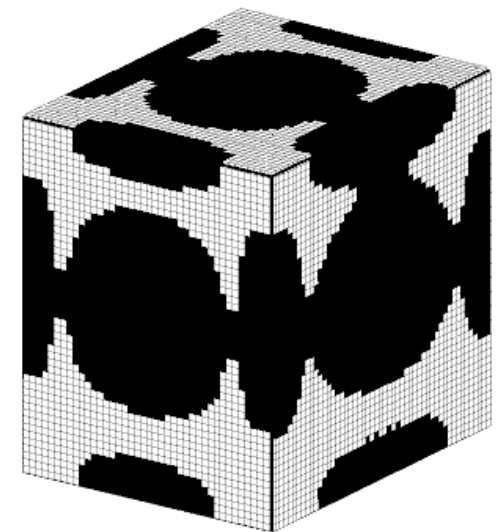

(b)

Fig. 11 Optimal layout of the damping material for the $1 \mathrm{st}-5$ th eigenmodes using the (a) proposed method and (b) conventional method.

Table 3 Loss factor differences between the proposed and conventional methods.

\begin{tabular}{lccc} 
& Proposed & Conventional & Difference [\%] \\
\hline Mode1 & 0.0633 & 0.0642 & -1.382 \\
Mode2 & 0.0635 & 0.0651 & -2.550 \\
Mode3 & 0.0647 & 0.0641 & 0.984 \\
Mode4 & 0.0628 & 0.0627 & 0.062 \\
Mode5 & 0.0666 & 0.0671 & -0.750 \\
\hline
\end{tabular}

\section{Conclusions}

This study proposes a method to linearize optimization problems using the assumption that the effect of design variables to mode shape is negligible when the base structure is damped with unconstrained layer damping treatment. The proposed method can obtain optimal configuration without iteration. The purpose of this study was to improve time efficiency for optimization of problems in optimal layout of damping material. Using this method, it was possible to create an optimal layout with only one eigenvalue analysis and optimization. The results are summarized below.

1. The loss factor after optimization was almost the same as that of the conventional method that requires iteration. No significant difference was found in the optimal layout.

2. Result of the verification on the validity of the assumption in the numerical example using MAC showed that the change of the mode shape before and after the optimization was extremely small. In other words, it was determined that the approximations made using the assumptions were valid and demonstrated the optimality of the original nonlinear programming problem.

3. The same result as that of the single-mode optimization was obtained even if optimization was performed for multiple modes, indicating that the proposed method could be applied to multiple modes.

\section{References}

Alok, S., Jaejong, P., Diana, C., and Michael, J. M., Experimental validation of 3D printed patient-specific implants using digital image correlation and finite element analysis, Computers in Biology and Medicine, Vol. 52 (2014), pp. 817.

Bendsøe, M. P. and Kikuchi, N., Generating optimal topologies in structural design using a homogenization method, Computer Methods in Applied Mechanics and Engineering, Vol. 71 (1988), pp. 197-224.

Bendsøe, M. P. and Sigmund, O., Material interpolation schemes in topology optimization, Archive of Applied Mechanics, Vol. 69 (1999), pp. $635-654$ 
Cameron, T., Glaucio, H. P. and Chau, H. Le., Honeycomb Wachspress finite elements for structural topology optimization, Structural and Multidisciplinary Optimization, Vol. 37 (2009), pp. 569-583.

$\mathrm{Du}$, J. and Olhoff, N., Minimization of sound radiation from vibrating bi-material structures using topology optimization, Struct Multidisc Optim, Vol. 33 (2007), pp. 305-321.

Ercan, M. D., Shailesh, N. J., and Feng, Z., Topology Optimization, Additive Layer Manufacturing, and Experimental Testing of an Air-Cooled Heat Sink, Journal of Mechanical Design, Vol. 137 (2015), 111403.

Johnson, C. and Kienholz, D., Finite element prediction of damping in structures with constrained viscoelastic layers, American Institute of Aeronautics and Astronautics Journal, Vol. 20 (1982), pp. 1284 - 1290.

Ling, Z., Rongle, X., Yi, W. and Sabbagh, A., Topology optimization of constrained layer damping on plates using Method of Moving Asymptote (MMA) approach, Shock and Vibration, Vol. 18 (2011), pp. 221 - 244.

Ma, Z., Kikuchi, N. and Cheng, H., Topological design for vibrating structures, Computer Methods in Applied Mechanics and Engineering, Vol. 121 (1995), pp. 259-280.

Pastor, M. and Binda, M., Modal Assurance Criterion, Procedia Engineering, Vol. 48 (2012), pp. 543-548.

Sigmund, O., Morphology-based black and white filters for topology optimization, Vol. 33 (2007), pp. 401-424.

Suzuki, K. and Kikuchi, N., A homogenization method for shape and topology optimization, Computer Methods in Applied Mechanics and Engineering, Vol. 93 (1991), pp. 291-318.

Wang, M., Wang, X. and Guo, D., A level set method for structural topology optimization, Computer Methods in Applied Mechanics and Engineering, Vol. 192 (2003), pp. 227-246.

Yamamoto, T., Yamada, T., Izui, K. and Nishiwaki, S., Topology optimization of free-layer damping material on a thin panel for maximizing modal loss factors expressed by only real eigenvalues, Journal of Sound and Vibration, Vol. 358 (2015), pp. 84-96.

Yang, R. and Chuang, C., Optimal topology design using linear programming, Computers \& Structures, Vol. 52 (1994), pp. $265-275$. 